\title{
Safety first portfolio choice based on financial and sustainability returns - Supplementary material
}

\author{
Gregor Dorfleitner, Sebastian Utz* \\ Department of Finance, University of Regensburg, 93040 Regensburg, Germany
}

\begin{abstract}
In addition to the theoretical and empirical deliberations in the associated paper, we display several figures and tables in this supplementary material.
\end{abstract}

\section{Appendix A. Data}

Before we outline any additional results we provide Table A.1 of the expected financial and sustainability returns, which we estimate as described in the paper for the ten subportfolios. Furthermore, we provide the covariance matrices of the financial return $\Sigma_{R}$, the sustainability return $\Sigma_{S R}$ and the covariance matrix between financial and sustainability returns $\Sigma_{R, S R}$. As shown in Table A.1 the rated companies are members of one of the ten common industrial sectors. As the number of companies in each sector differs, we pick ten companies from each sector in account of the estimation described in the paper. The indices of the covariance matrices displayed in this section is of the same order as those displaying the sectors in Table A.1.

\footnotetext{
*Corresponding author. Tel. +49 9416309158 26; fax +49 9419434608.

Email addresses: gregor.dorfleitner@wiwi.uni-r.de (Gregor Dorfleitner), sebastian.utz@wiwi.uni-r.de (Sebastian Utz)
} 
Table A.1: Expected annual financial and sustainability returns of each subportfolio.

\begin{tabular}{lrr}
\hline Industrial sector & $\mathbf{E}\left[R_{j}\right]$ & $\mathbf{E}\left[S R_{j}\right]$ \\
\hline Financials & 0.0802 & 0.1276 \\
Industrials & 0.1437 & 0.1305 \\
Utilities & 0.0915 & 0.1188 \\
Health Care & 0.0541 & 0.0749 \\
Consumer Discretionary & 0.1991 & 0.0644 \\
Information Technology & 0.0956 & 0.0696 \\
Energy & 0.1061 & 0.1013 \\
Consumer Staples & 0.0878 & 0.1340 \\
Telecommunication Services & 0.0726 & 0.1270 \\
Materials & 0.1467 & 0.1293 \\
\hline
\end{tabular}

$\Sigma_{R}=\left(\begin{array}{llllllllll}0.1455 & 0.0745 & 0.0677 & 0.0446 & 0.0684 & 0.0570 & 0.0702 & 0.0533 & 0.0425 & 0.0855 \\ 0.0745 & 0.0699 & 0.0471 & 0.0263 & 0.0433 & 0.0385 & 0.0530 & 0.0333 & 0.0332 & 0.0670 \\ 0.0677 & 0.0471 & 0.0491 & 0.0262 & 0.0382 & 0.0337 & 0.0477 & 0.0317 & 0.0303 & 0.0551 \\ 0.0446 & 0.0263 & 0.0262 & 0.0273 & 0.0256 & 0.0218 & 0.0266 & 0.0235 & 0.0183 & 0.0305 \\ 0.0684 & 0.0433 & 0.0382 & 0.0256 & 0.0540 & 0.0316 & 0.0424 & 0.0257 & 0.0263 & 0.0540 \\ 0.0570 & 0.0385 & 0.0337 & 0.0218 & 0.0316 & 0.0577 & 0.0380 & 0.0289 & 0.0307 & 0.0419 \\ 0.0702 & 0.0530 & 0.0477 & 0.0266 & 0.0424 & 0.0380 & 0.0737 & 0.0318 & 0.0314 & 0.0583 \\ 0.0533 & 0.0333 & 0.0317 & 0.0235 & 0.0257 & 0.0289 & 0.0318 & 0.0394 & 0.0278 & 0.0377 \\ 0.0425 & 0.0332 & 0.0303 & 0.0183 & 0.0263 & 0.0307 & 0.0314 & 0.0278 & 0.0346 & 0.0403 \\ 0.0855 & 0.0670 & 0.0551 & 0.0305 & 0.0540 & 0.0419 & 0.0583 & 0.0377 & 0.0403 & 0.0868\end{array}\right)$

$\Sigma_{S R}=\left(\begin{array}{llllllllll}0.0057 & 0.0047 & 0.0039 & 0.0033 & 0.0045 & 0.0036 & 0.0034 & 0.0043 & 0.0036 & 0.0048 \\ 0.0047 & 0.0042 & 0.0033 & 0.0028 & 0.0039 & 0.0032 & 0.0030 & 0.0037 & 0.0031 & 0.0041 \\ 0.0039 & 0.0033 & 0.0028 & 0.0021 & 0.0031 & 0.0024 & 0.0023 & 0.0029 & 0.0025 & 0.0033 \\ 0.0033 & 0.0028 & 0.0021 & 0.0021 & 0.0027 & 0.0023 & 0.0021 & 0.0027 & 0.0021 & 0.0029 \\ 0.0045 & 0.0039 & 0.0031 & 0.0027 & 0.0038 & 0.0030 & 0.0028 & 0.0035 & 0.0029 & 0.0040 \\ 0.0036 & 0.0032 & 0.0024 & 0.0023 & 0.0030 & 0.0026 & 0.0024 & 0.0030 & 0.0024 & 0.0032 \\ 0.0034 & 0.0030 & 0.0023 & 0.0021 & 0.0028 & 0.0024 & 0.0022 & 0.0028 & 0.0022 & 0.0030 \\ 0.0043 & 0.0037 & 0.0029 & 0.0027 & 0.0035 & 0.0030 & 0.0028 & 0.0034 & 0.0028 & 0.0038 \\ 0.0036 & 0.0031 & 0.0025 & 0.0021 & 0.0029 & 0.0024 & 0.0022 & 0.0028 & 0.0024 & 0.0031 \\ 0.0048 & 0.0041 & 0.0033 & 0.0029 & 0.0040 & 0.0032 & 0.0030 & 0.0038 & 0.0031 & 0.0043\end{array}\right)$ 
$\Sigma_{R, S R}=\left(\begin{array}{cccccccccc}-0.0153 & -0.0104 & -0.0099 & -0.0106 & -0.0133 & -0.0088 & -0.0077 & -0.0113 & -0.0087 & -0.0141 \\ -0.0052 & -0.0021 & -0.0028 & -0.0048 & -0.0046 & -0.0027 & -0.0020 & -0.0039 & -0.0024 & -0.0050 \\ -0.0083 & -0.0059 & -0.0047 & -0.0069 & -0.0068 & -0.0060 & -0.0052 & -0.0072 & -0.0049 & -0.0074 \\ -0.0024 & -0.0021 & -0.0009 & -0.0035 & -0.0033 & -0.0028 & -0.0021 & -0.0032 & -0.0013 & -0.0036 \\ 0.0013 & 0.0035 & 0.0016 & -0.0007 & 0.0013 & 0.0017 & 0.0020 & 0.0014 & 0.0017 & 0.0014 \\ -0.0075 & -0.0050 & -0.0039 & -0.0066 & -0.0049 & -0.0056 & -0.0050 & -0.0066 & -0.0044 & -0.0057 \\ -0.0119 & -0.0075 & -0.0076 & -0.0079 & -0.0090 & -0.0066 & -0.0059 & -0.0085 & -0.0068 & -0.0097 \\ -0.0050 & -0.0031 & -0.0024 & -0.0051 & -0.0041 & -0.0039 & -0.0032 & -0.0047 & -0.0027 & -0.0046 \\ -0.0035 & -0.0025 & -0.0013 & -0.0046 & -0.0030 & -0.0036 & -0.0029 & -0.0041 & -0.0019 & -0.0036 \\ -0.0033 & -0.0006 & -0.0013 & -0.0044 & -0.0034 & -0.0019 & -0.0011 & -0.0029 & -0.0011 & -0.0038\end{array}\right)$

The negative covariances of the expected annual financial and sustainability returns (as shown in Table A.1 and $\Sigma_{R, S R}$ ) are consistent with Hong \& Kacperczyk (2009) and Fabozzi et al. (2008), who find that sin stock portfolios yield higher financial returns than SRI portfolios. Hence, all other subportfolios except one show negative correlations between financial and sustainability returns in our sample, and the portfolio choice with financial and sustainable objective values contains a real tradeoff between financial and sustainable returns.

\section{Appendix B. Efficient frontier}

As the feasible sets of the joint distribution model (JDM) and the marginal distributions model (MDM) are independent of $\gamma$, we find an efficient frontier for both models in the $\mathbf{E}\left[w^{\prime} R\right]-\mathbf{E}\left[w^{\prime} S R\right]$-space, which is displayed in Figure B.1. Dependent on the preference parameter $\gamma$, all optimal solutions are contained in the black border displayed in Figure B.1. This is due to the fact that the used preference functional is a convex combination with positive weights on both objective values (expected financial and expected sustainability return).

\section{Appendix C. Joint versus marginal distributions model}

In the paper we also show that the probability constraint of the MDM is more restrictive under the conditions of positive quadrant dependence compared with the probability constraints of the JDM. Figure C.2 illustrates the 
Figure B.1: Efficient frontier of the JDM and the MDM and the convex hull of the feasible sets for an investor with $c_{R}=-0.15, c_{S R}=-0.02, \alpha=0.13$ respectively $\alpha_{R}=0.094$ and $\alpha_{S R}=0.04$ and the restriction $0 \leq w_{i} \leq 0.25$ for all $i$ on the portfolio weights based on the financial and sustainability returns displayed in Table A.1.

Efficient frontier Joint distribution model

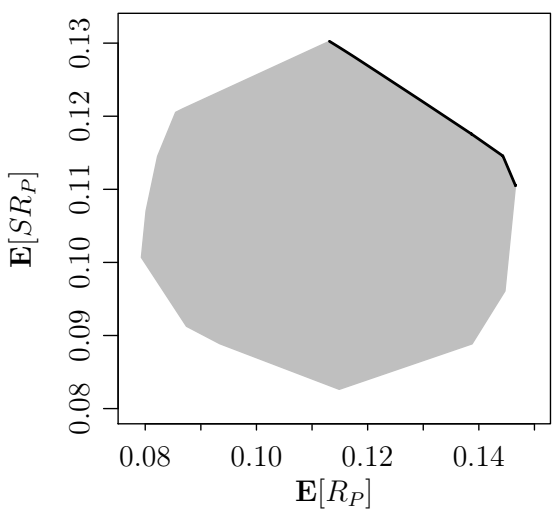

Efficient frontier Marginal distributions model

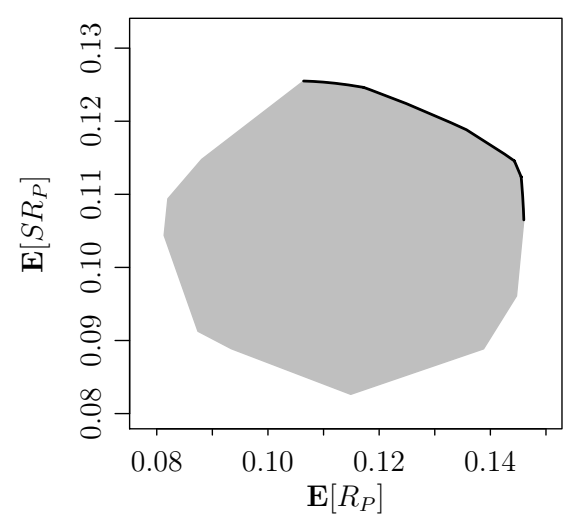

relation between the objective values of the JDM and the MDM for various preferences $\gamma$.

Figure C.2: Optimal objective function values of the JDM and the MDM dependent on $c_{S R} \in[-0.02,0.026]$ and $\gamma \in\{0,0.1,0.5,0.9\}$. Calculations are based on the parameter specification used in section 5.2 of the associated paper.
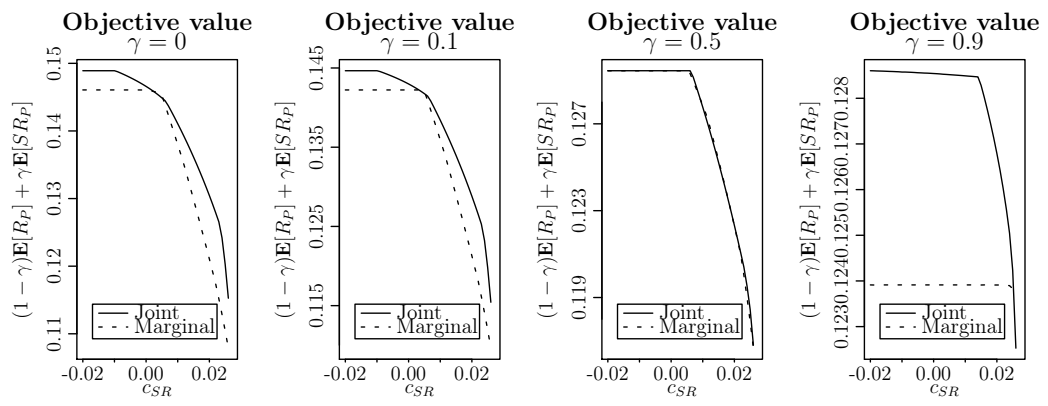


\section{Appendix D. Model comparison with respect to the default prob- abilities}

Additionally, we expose the three models to a simple test: We generate 100 random vectors of financial and sustainability returns using the distributions of the optimal portfolios and display the tuples of returns for all three models in Figure D.3. It is obvious that the JDM and the MDM yield

Figure D.3: Illustration of tuples $(R, S R)$ for optimal portfolios for 100 randomly generated returns. The calculations are based on the data used in section 5.2 of the associated paper.
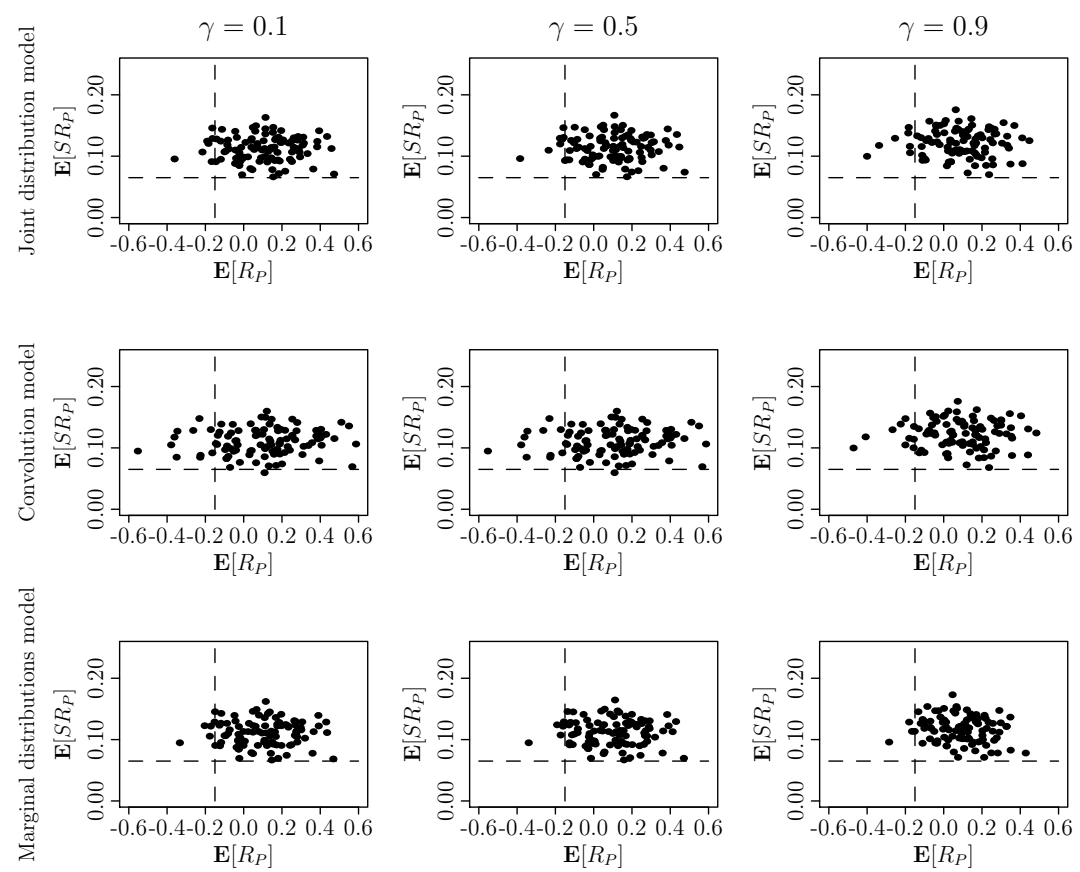

good solutions for the presented portfolio problem. The returns exceed the thresholds in almost $1-\alpha$ of the cases for the JDM and in $1-\alpha_{R}$ respectively $1-\alpha_{S R}$ of the cases in all of the both dimensions for the MDM. Although we scale financial and sustainability return the same size in expected values, the sample range which is created by the CM produces strange yet model consistent results. Due to the convex combination of both returns and even 
of both thresholds it is not the primary aim of the CM to ensure a default in one or both dimensions on their own.

Additionally, we observe confirmation of our results discussing the objective value and the financial and sustainability returns for varying $\gamma$. With increasing $\gamma$, the cloud of return tuples moves up; therewith, a sustainable loss is less likely and one can claim that an increasing threshold $c_{S R}$ does not affect the optimal solution in a certain segment.

\section{Appendix E. Portfolio weights}

The Tables E.2 to E.12 display the change of portfolio weights of the optimal solutions of the three different GSFI models in several parameter setups. However, we linearly transform the expected sustainability return into the same range as that of the expected financial return, while the standard deviations have different ranges. Due to this fact, the CM generates curious outcomes for several parameter setups with ranges, which are too varied.

Table E.2: Portfolio weights of the optimal solutions of the JDM with $\gamma=0$ in percent.

\begin{tabular}{ccccccccc}
\hline$c_{S R}$ & -0.020 & -0.013 & -0.006 & 0.001 & 0.008 & 0.015 & 0.022 & 0.026 \\
\hline$w_{1}$ & 0.0 & 0.0 & 0.0 & 0.0 & 0.0 & 0.0 & 0.0 & 0.0 \\
$w_{2}$ & 25.0 & 25.0 & 25.0 & 25.0 & 25.0 & 25.0 & 25.0 & 18.3 \\
$w_{3}$ & 0.0 & 0.0 & 0.0 & 0.0 & 0.0 & 0.0 & 0.0 & 13.2 \\
$w_{4}$ & 0.0 & 0.0 & 0.0 & 0.0 & 0.0 & 0.0 & 0.0 & 0.0 \\
$w_{5}$ & 25.0 & 25.0 & 25.0 & 25.0 & 25.0 & 25.0 & 25.0 & 18.5 \\
$w_{6}$ & 0.0 & 0.0 & 0.0 & 0.0 & 0.0 & 0.0 & 0.0 & 0.0 \\
$w_{7}$ & 25.0 & 25.0 & 20.7 & 10.7 & 0.0 & 0.0 & 0.0 & 0.0 \\
$w_{8}$ & 0.0 & 0.0 & 4.3 & 14.3 & 25.0 & 25.0 & 25.0 & 25.0 \\
$w_{9}$ & 0.0 & 0.0 & 0.0 & 0.0 & 2.0 & 11.0 & 22.2 & 25.0 \\
$w_{10}$ & 25.0 & 25.0 & 25.0 & 25.0 & 23.0 & 14.0 & 2.8 & 0.0 \\
\hline$\mu_{R}$ & 0.149 & 0.149 & 0.148 & 0.146 & 0.143 & 0.136 & 0.128 & 0.115 \\
$\sigma_{R}$ & 0.247 & 0.247 & 0.243 & 0.236 & 0.226 & 0.213 & 0.198 & 0.186 \\
$\mu_{S R}$ & 0.106 & 0.106 & 0.108 & 0.111 & 0.115 & 0.114 & 0.114 & 0.117 \\
$\sigma_{S R}$ & 0.028 & 0.028 & 0.028 & 0.029 & 0.029 & 0.028 & 0.028 & 0.027 \\
\hline
\end{tabular}


Table E.3: Portfolio weights of the optimal solutions of the JDM with $\gamma=0.1$ in percent.

\begin{tabular}{lcccccccc}
\hline$c_{S R}$ & -0.020 & -0.013 & -0.006 & 0.001 & 0.008 & 0.015 & 0.022 & 0.026 \\
\hline$w_{1}$ & 0.0 & 0.0 & 0.0 & 0.0 & 0.0 & 0.0 & 0.0 & 0.0 \\
$w_{2}$ & 25.0 & 25.0 & 25.0 & 25.0 & 25.0 & 25.0 & 25.0 & 19.1 \\
$w_{3}$ & 0.0 & 0.0 & 0.0 & 0.0 & 0.0 & 0.0 & 0.0 & 12.8 \\
$w_{4}$ & 0.0 & 0.0 & 0.0 & 0.0 & 0.0 & 0.0 & 0.0 & 0.0 \\
$w_{5}$ & 25.0 & 25.0 & 25.0 & 25.0 & 25.0 & 25.0 & 25.0 & 18.1 \\
$w_{6}$ & 0.0 & 0.0 & 0.0 & 0.0 & 0.0 & 0.0 & 0.0 & 0.0 \\
$w_{7}$ & 25.0 & 25.0 & 20.7 & 10.7 & 0.0 & 0.0 & 0.0 & 0.0 \\
$w_{8}$ & 0.0 & 0.0 & 4.3 & 14.3 & 25.0 & 25.0 & 25.0 & 25.0 \\
$w_{9}$ & 0.0 & 0.0 & 0.0 & 0.0 & 2.0 & 11.0 & 22.2 & 25.0 \\
$w_{10}$ & 25.0 & 25.0 & 25.0 & 25.0 & 23.0 & 14.0 & 2.8 & 0.0 \\
\hline$\mu_{R}$ & 0.149 & 0.149 & 0.148 & 0.146 & 0.143 & 0.136 & 0.128 & 0.115 \\
$\sigma_{R}$ & 0.247 & 0.247 & 0.243 & 0.236 & 0.226 & 0.213 & 0.198 & 0.186 \\
$\mu_{S R}$ & 0.106 & 0.106 & 0.108 & 0.111 & 0.115 & 0.114 & 0.114 & 0.117 \\
$\sigma_{S R}$ & 0.028 & 0.028 & 0.028 & 0.029 & 0.029 & 0.028 & 0.028 & 0.027 \\
\hline
\end{tabular}

Table E.4: Portfolio weights of the optimal solutions of the JDM with $\gamma=0.9$ in percent.

\begin{tabular}{lcccccccc}
\hline$c_{S R}$ & -0.020 & -0.013 & -0.006 & 0.001 & 0.008 & 0.015 & 0.022 & 0.026 \\
\hline$w_{1}$ & 10.4 & 9.0 & 7.4 & 5.3 & 2.8 & 0.0 & 0.0 & 0.0 \\
$w_{2}$ & 25.0 & 25.0 & 25.0 & 25.0 & 25.0 & 25.0 & 25.0 & 23.1 \\
$w_{3}$ & 0.0 & 0.0 & 0.0 & 0.0 & 0.0 & 1.2 & 16.3 & 22.0 \\
$w_{4}$ & 0.0 & 0.0 & 0.0 & 0.0 & 0.0 & 0.0 & 0.0 & 0.0 \\
$w_{5}$ & 0.0 & 0.0 & 0.0 & 0.0 & 0.0 & 0.0 & 0.0 & 4.9 \\
$w_{6}$ & 0.0 & 0.0 & 0.0 & 0.0 & 0.0 & 0.0 & 0.0 & 0.0 \\
$w_{7}$ & 0.0 & 0.0 & 0.0 & 0.0 & 0.0 & 0.0 & 0.0 & 0.0 \\
$w_{8}$ & 25.0 & 25.0 & 25.0 & 25.0 & 25.0 & 25.0 & 25.0 & 25.0 \\
$w_{9}$ & 14.6 & 16.0 & 17.6 & 19.7 & 22.2 & 25.0 & 25.0 & 25.0 \\
$w_{10}$ & 25.0 & 25.0 & 25.0 & 25.0 & 25.0 & 23.8 & 8.7 & 0.0 \\
\hline$\mu_{R}$ & 0.113 & 0.113 & 0.113 & 0.113 & 0.113 & 0.112 & 0.104 & 0.103 \\
$\sigma_{R}$ & 0.230 & 0.228 & 0.225 & 0.222 & 0.218 & 0.212 & 0.193 & 0.184 \\
$\mu_{S R}$ & 0.130 & 0.130 & 0.130 & 0.130 & 0.130 & 0.130 & 0.128 & 0.125 \\
$\sigma_{S R}$ & 0.031 & 0.031 & 0.030 & 0.030 & 0.030 & 0.030 & 0.029 & 0.028 \\
\hline
\end{tabular}


Table E.5: Portfolio weights of the optimal solutions of the CM with $\gamma=0$ in percent.

\begin{tabular}{lcccccccc}
\hline$c_{S R}$ & -0.020 & -0.013 & -0.006 & 0.001 & 0.008 & 0.015 & 0.022 & 0.026 \\
\hline$w_{1}$ & 0.0 & 0.0 & 0.0 & 0.0 & 0.0 & 0.0 & 0.0 & 0.0 \\
$w_{2}$ & 14.7 & 14.7 & 14.7 & 14.7 & 14.7 & 14.7 & 14.7 & 14.7 \\
$w_{3}$ & 0.0 & 0.0 & 0.0 & 0.0 & 0.0 & 0.0 & 0.0 & 0.0 \\
$w_{4}$ & 17.2 & 17.2 & 17.2 & 17.2 & 17.2 & 17.2 & 17.2 & 17.2 \\
$w_{5}$ & 25.0 & 25.0 & 25.0 & 25.0 & 25.0 & 25.0 & 25.0 & 25.0 \\
$w_{6}$ & 7.4 & 7.4 & 7.4 & 7.4 & 7.4 & 7.4 & 7.4 & 7.4 \\
$w_{7}$ & 0.0 & 0.0 & 0.0 & 0.0 & 0.0 & 0.0 & 0.0 & 0.0 \\
$w_{8}$ & 23.1 & 23.1 & 23.1 & 23.1 & 23.1 & 23.1 & 23.1 & 23.1 \\
$w_{9}$ & 12.6 & 12.6 & 12.6 & 12.6 & 12.6 & 12.6 & 12.6 & 12.6 \\
$w_{10}$ & 0.0 & 0.0 & 0.0 & 0.0 & 0.0 & 0.0 & 0.0 & 0.0 \\
\hline$\mu_{R}$ & 0.117 & 0.117 & 0.117 & 0.117 & 0.117 & 0.117 & 0.117 & 0.117 \\
$\sigma_{R}$ & 0.189 & 0.189 & 0.189 & 0.189 & 0.189 & 0.189 & 0.189 & 0.189 \\
$\mu_{S R}$ & 0.100 & 0.100 & 0.100 & 0.100 & 0.100 & 0.100 & 0.100 & 0.100 \\
$\sigma_{S R}$ & 0.028 & 0.028 & 0.028 & 0.028 & 0.028 & 0.028 & 0.028 & 0.028 \\
\hline
\end{tabular}

Table E.6: Portfolio weights of the optimal solutions of the CM with $\gamma=0.1$ in percent.

\begin{tabular}{lcccccccc}
\hline$c_{S R}$ & -0.020 & -0.013 & -0.006 & 0.001 & 0.008 & 0.015 & 0.022 & 0.026 \\
\hline$w_{1}$ & 0.0 & 0.0 & 0.0 & 0.0 & 0.0 & 0.0 & 0.0 & 0.0 \\
$w_{2}$ & 25.0 & 25.0 & 25.0 & 25.0 & 25.0 & 25.0 & 25.0 & 25.0 \\
$w_{3}$ & 0.0 & 0.0 & 0.0 & 0.0 & 0.0 & 0.0 & 0.0 & 0.0 \\
$w_{4}$ & 0.0 & 0.0 & 0.0 & 0.0 & 0.0 & 0.0 & 0.0 & 0.0 \\
$w_{5}$ & 25.0 & 25.0 & 25.0 & 25.0 & 25.0 & 25.0 & 25.0 & 25.0 \\
$w_{6}$ & 11.4 & 11.3 & 11.2 & 11.0 & 10.9 & 10.8 & 10.7 & 10.6 \\
$w_{7}$ & 0.0 & 0.0 & 0.0 & 0.0 & 0.0 & 0.0 & 0.0 & 0.0 \\
$w_{8}$ & 25.0 & 25.0 & 25.0 & 25.0 & 25.0 & 25.0 & 25.0 & 25.0 \\
$w_{9}$ & 6.3 & 7.2 & 8.0 & 8.9 & 9.8 & 10.7 & 11.6 & 12.2 \\
$w_{10}$ & 7.3 & 6.6 & 5.8 & 5.1 & 4.3 & 3.5 & 2.7 & 2.2 \\
\hline$\mu_{R}$ & 0.134 & 0.133 & 0.133 & 0.132 & 0.131 & 0.131 & 0.130 & 0.130 \\
$\sigma_{R}$ & 0.211 & 0.210 & 0.209 & 0.208 & 0.207 & 0.206 & 0.205 & 0.204 \\
$\mu_{S R}$ & 0.108 & 0.108 & 0.108 & 0.108 & 0.108 & 0.108 & 0.108 & 0.108 \\
$\sigma_{S R}$ & 0.028 & 0.028 & 0.028 & 0.028 & 0.028 & 0.028 & 0.027 & 0.027 \\
\hline
\end{tabular}


Table E.7: Portfolio weights of the optimal solutions of the CM with $\gamma=0.5$ in percent.

\begin{tabular}{lcccccccc}
\hline$c_{S R}$ & -0.020 & -0.013 & -0.006 & 0.001 & 0.008 & 0.015 & 0.022 & 0.026 \\
\hline$w_{1}$ & 0.0 & 0.0 & 0.0 & 0.0 & 0.0 & 0.0 & 0.0 & 0.0 \\
$w_{2}$ & 25.0 & 25.0 & 25.0 & 25.0 & 25.0 & 25.0 & 25.0 & 25.0 \\
$w_{3}$ & 0.0 & 0.0 & 0.0 & 0.0 & 0.0 & 0.0 & 0.0 & 0.0 \\
$w_{4}$ & 0.0 & 0.0 & 0.0 & 0.0 & 0.0 & 0.0 & 0.0 & 0.0 \\
$w_{5}$ & 25.0 & 25.0 & 25.0 & 25.0 & 25.0 & 25.0 & 25.0 & 25.0 \\
$w_{6}$ & 0.0 & 0.0 & 0.0 & 0.0 & 0.0 & 0.0 & 0.0 & 0.0 \\
$w_{7}$ & 0.0 & 0.0 & 0.0 & 0.0 & 0.0 & 0.0 & 0.0 & 0.0 \\
$w_{8}$ & 25.0 & 25.0 & 25.0 & 25.0 & 25.0 & 25.0 & 25.0 & 25.0 \\
$w_{9}$ & 0.0 & 0.0 & 0.0 & 0.0 & 0.0 & 0.0 & 0.0 & 0.0 \\
$w_{10}$ & 25.0 & 25.0 & 25.0 & 25.0 & 25.0 & 25.0 & 25.0 & 25.0 \\
\hline$\mu_{R}$ & 0.144 & 0.144 & 0.144 & 0.144 & 0.144 & 0.144 & 0.144 & 0.144 \\
$\sigma_{R}$ & 0.229 & 0.229 & 0.229 & 0.229 & 0.229 & 0.229 & 0.229 & 0.229 \\
$\mu_{S R}$ & 0.115 & 0.115 & 0.115 & 0.115 & 0.115 & 0.115 & 0.115 & 0.115 \\
$\sigma_{S R}$ & 0.029 & 0.029 & 0.029 & 0.029 & 0.029 & 0.029 & 0.029 & 0.029 \\
\hline
\end{tabular}

Table E.8: Portfolio weights of the optimal solutions of the CM with $\gamma=0.9$ in percent.

\begin{tabular}{lcccccccc}
\hline$c_{S R}$ & -0.020 & -0.013 & -0.006 & 0.001 & 0.008 & 0.015 & 0.022 & 0.026 \\
\hline$w_{1}$ & 25.0 & 25.0 & 25.0 & 25.0 & 25.0 & 25.0 & 25.0 & 25.0 \\
$w_{2}$ & 25.0 & 25.0 & 25.0 & 25.0 & 25.0 & 25.0 & 25.0 & 25.0 \\
$w_{3}$ & 0.0 & 0.0 & 0.0 & 0.0 & 0.0 & 0.0 & 0.0 & 0.0 \\
$w_{4}$ & 0.0 & 0.0 & 0.0 & 0.0 & 0.0 & 0.0 & 0.0 & 0.0 \\
$w_{5}$ & 0.0 & 0.0 & 0.0 & 0.0 & 0.0 & 0.0 & 0.0 & 0.0 \\
$w_{6}$ & 0.0 & 0.0 & 0.0 & 0.0 & 0.0 & 0.0 & 0.0 & 0.0 \\
$w_{7}$ & 0.0 & 0.0 & 0.0 & 0.0 & 0.0 & 0.0 & 0.0 & 0.0 \\
$w_{8}$ & 25.0 & 25.0 & 25.0 & 25.0 & 25.0 & 25.0 & 25.0 & 25.0 \\
$w_{9}$ & 0.0 & 0.0 & 0.0 & 0.0 & 0.0 & 0.0 & 0.0 & 0.0 \\
$w_{10}$ & 25.0 & 25.0 & 25.0 & 25.0 & 25.0 & 25.0 & 25.0 & 25.0 \\
\hline$\mu_{R}$ & 0.115 & 0.115 & 0.115 & 0.115 & 0.115 & 0.115 & 0.115 & 0.115 \\
$\sigma_{R}$ & 0.256 & 0.256 & 0.256 & 0.256 & 0.256 & 0.256 & 0.256 & 0.256 \\
$\mu_{S R}$ & 0.130 & 0.130 & 0.130 & 0.130 & 0.130 & 0.130 & 0.130 & 0.130 \\
$\sigma_{S R}$ & 0.032 & 0.032 & 0.032 & 0.032 & 0.032 & 0.032 & 0.032 & 0.032 \\
\hline
\end{tabular}


Table E.9: Portfolio weights of the optimal solutions of the MDM with $\gamma=0$ in percent. It is due to rounding errors that the weights do not amount to 100 in all cases.

\begin{tabular}{lcccccccc}
\hline$c_{S R}$ & -0.020 & -0.013 & -0.006 & 0.001 & 0.008 & 0.015 & 0.022 & 0.026 \\
\hline$w_{1}$ & 0.0 & 0.0 & 0.0 & 0.0 & 0.0 & 0.0 & 0.0 & 0.0 \\
$w_{2}$ & 25.0 & 25.0 & 25.0 & 25.0 & 25.0 & 25.0 & 25.0 & 25.0 \\
$w_{3}$ & 0.0 & 0.0 & 0.0 & 0.0 & 4.8 & 0.0 & 0.0 & 13.6 \\
$w_{4}$ & 0.0 & 0.0 & 0.0 & 0.0 & 0.0 & 0.0 & 0.0 & 0.0 \\
$w_{5}$ & 25.0 & 25.0 & 25.0 & 25.0 & 22.6 & 15.5 & 8.7 & 5.1 \\
$w_{6}$ & 14.3 & 14.3 & 14.3 & 7.9 & 0.0 & 0.0 & 0.0 & 0.0 \\
$w_{7}$ & 3.4 & 3.4 & 3.4 & 5.6 & 0.2 & 0.0 & 0.0 & 0.0 \\
$w_{8}$ & 7.2 & 7.2 & 7.3 & 11.5 & 16.6 & 19.6 & 24.8 & 25.0 \\
$w_{9}$ & 0.0 & 0.0 & 0.0 & 0.0 & 5.9 & 17.1 & 25.0 & 25.0 \\
$w_{10}$ & 25.0 & 25.0 & 25.0 & 25.0 & 25.0 & 22.8 & 16.5 & 6.3 \\
\hline$\mu_{R}$ & 0.146 & 0.146 & 0.146 & 0.146 & 0.141 & 0.130 & 0.117 & 0.108 \\
$\sigma_{R}$ & 0.241 & 0.241 & 0.241 & 0.237 & 0.227 & 0.219 & 0.207 & 0.192 \\
$\mu_{S R}$ & 0.104 & 0.104 & 0.104 & 0.108 & 0.115 & 0.120 & 0.125 & 0.125 \\
$\sigma_{S R}$ & 0.029 & 0.029 & 0.029 & 0.029 & 0.029 & 0.029 & 0.029 & 0.028 \\
\hline & & & & & & & &
\end{tabular}

Table E.10: Portfolio weights of the optimal solutions of the MDM with $\gamma=0.1$ in percent. It is due to rounding errors that the weights do not amount to 100 in all cases.

\begin{tabular}{lcccccccc}
\hline$c_{S R}$ & -0.020 & -0.013 & -0.006 & 0.001 & 0.008 & 0.015 & 0.022 & 0.026 \\
\hline$w_{1}$ & 0.0 & 0.0 & 0.0 & 0.0 & 0.0 & 0.0 & 0.0 & 0.0 \\
$w_{2}$ & 25.0 & 25.0 & 25.0 & 25.0 & 25.0 & 25.0 & 25.0 & 25.0 \\
$w_{3}$ & 0.0 & 0.0 & 0.0 & 0.0 & 3.2 & 0.0 & 0.0 & 13.6 \\
$w_{4}$ & 0.0 & 0.0 & 0.0 & 0.0 & 0.0 & 0.0 & 0.0 & 0.0 \\
$w_{5}$ & 25.0 & 25.0 & 25.0 & 25.0 & 21.8 & 15.1 & 8.7 & 5.1 \\
$w_{6}$ & 1.1 & 1.1 & 1.1 & 1.0 & 0.0 & 0.0 & 0.0 & 0.0 \\
$w_{7}$ & 6.6 & 6.6 & 6.6 & 6.6 & 0.0 & 0.0 & 0.0 & 0.0 \\
$w_{8}$ & 17.3 & 17.3 & 17.3 & 17.4 & 24.9 & 23.6 & 25.0 & 25.0 \\
$w_{9}$ & 0.0 & 0.0 & 0.0 & 0.0 & 0.0 & 13.6 & 24.9 & 25.0 \\
$w_{10}$ & 25.0 & 25.0 & 25.0 & 25.0 & 25.0 & 22.7 & 16.5 & 6.3 \\
\hline$\mu_{R}$ & 0.146 & 0.146 & 0.146 & 0.146 & 0.141 & 0.130 & 0.117 & 0.108 \\
$\sigma_{R}$ & 0.234 & 0.234 & 0.234 & 0.234 & 0.227 & 0.219 & 0.207 & 0.192 \\
$\mu_{S R}$ & 0.112 & 0.112 & 0.112 & 0.112 & 0.116 & 0.121 & 0.125 & 0.125 \\
$\sigma_{S R}$ & 0.029 & 0.029 & 0.029 & 0.029 & 0.029 & 0.029 & 0.029 & 0.028 \\
\hline
\end{tabular}


Table E.11: Portfolio weights of the optimal solutions of the MDM with $\gamma=0.5$ in percent. It is due to rounding errors that the weights do not amount to 100 in all cases.

\begin{tabular}{lcccccccc}
\hline$c_{S R}$ & -0.020 & -0.013 & -0.006 & 0.001 & 0.008 & 0.015 & 0.022 & 0.026 \\
\hline$w_{1}$ & 0.0 & 0.0 & 0.0 & 0.0 & 0.0 & 0.0 & 0.0 & 0.0 \\
$w_{2}$ & 25.0 & 25.0 & 25.0 & 25.0 & 25.0 & 25.0 & 25.0 & 25.0 \\
$w_{3}$ & 0.0 & 0.0 & 0.0 & 0.0 & 3.2 & 0.0 & 0.0 & 13.6 \\
$w_{4}$ & 0.0 & 0.0 & 0.0 & 0.0 & 0.0 & 0.0 & 0.0 & 0.0 \\
$w_{5}$ & 25.0 & 25.0 & 25.0 & 25.0 & 21.8 & 14.9 & 8.7 & 5.1 \\
$w_{6}$ & 0.0 & 0.0 & 0.0 & 0.0 & 0.0 & 0.0 & 0.0 & 0.0 \\
$w_{7}$ & 0.0 & 0.0 & 0.0 & 0.0 & 0.0 & 0.0 & 0.0 & 0.0 \\
$w_{8}$ & 25.0 & 25.0 & 25.0 & 25.0 & 25.0 & 25.0 & 25.0 & 25.0 \\
$w_{9}$ & 0.0 & 0.0 & 0.0 & 0.0 & 0.0 & 12.4 & 24.8 & 25.0 \\
$w_{10}$ & 25.0 & 25.0 & 25.0 & 25.0 & 25.0 & 22.7 & 16.5 & 6.3 \\
\hline$\mu_{R}$ & 0.144 & 0.144 & 0.144 & 0.144 & 0.141 & 0.130 & 0.117 & 0.108 \\
$\sigma_{R}$ & 0.229 & 0.229 & 0.229 & 0.229 & 0.227 & 0.219 & 0.207 & 0.192 \\
$\mu_{S R}$ & 0.115 & 0.115 & 0.115 & 0.115 & 0.116 & 0.121 & 0.125 & 0.125 \\
$\sigma_{S R}$ & 0.029 & 0.029 & 0.029 & 0.029 & 0.029 & 0.029 & 0.029 & 0.028 \\
\hline
\end{tabular}

Table E.12: Portfolio weights of the optimal solutions of the MDM with $\gamma=0.9$ in percent.

\begin{tabular}{ccccccccc}
\hline$c_{S R}$ & -0.020 & -0.013 & -0.006 & 0.001 & 0.008 & 0.015 & 0.022 & 0.026 \\
\hline$w_{1}$ & 0.0 & 0.0 & 0.0 & 0.0 & 0.0 & 0.0 & 0.0 & 0.0 \\
$w_{2}$ & 25.0 & 25.0 & 25.0 & 25.0 & 25.0 & 25.0 & 25.0 & 25.0 \\
$w_{3}$ & 3.5 & 3.5 & 3.5 & 3.5 & 3.5 & 3.5 & 3.5 & 13.6 \\
$w_{4}$ & 0.0 & 0.0 & 0.0 & 0.0 & 0.0 & 0.0 & 0.0 & 0.0 \\
$w_{5}$ & 7.6 & 7.6 & 7.6 & 7.6 & 7.6 & 7.6 & 7.6 & 5.1 \\
$w_{6}$ & 0.0 & 0.0 & 0.0 & 0.0 & 0.0 & 0.0 & 0.0 & 0.0 \\
$w_{7}$ & 0.0 & 0.0 & 0.0 & 0.0 & 0.0 & 0.0 & 0.0 & 0.0 \\
$w_{8}$ & 25.0 & 25.0 & 25.0 & 25.0 & 25.0 & 25.0 & 25.0 & 25.0 \\
$w_{9}$ & 25.0 & 25.0 & 25.0 & 25.0 & 25.0 & 25.0 & 25.0 & 25.0 \\
$w_{10}$ & 13.9 & 13.9 & 13.9 & 13.9 & 13.9 & 13.9 & 13.9 & 6.3 \\
\hline$\mu_{R}$ & 0.115 & 0.115 & 0.115 & 0.115 & 0.115 & 0.115 & 0.115 & 0.108 \\
$\sigma_{R}$ & 0.203 & 0.203 & 0.203 & 0.203 & 0.203 & 0.203 & 0.203 & 0.192 \\
$\mu_{S R}$ & 0.125 & 0.125 & 0.125 & 0.125 & 0.125 & 0.125 & 0.125 & 0.125 \\
$\sigma_{S R}$ & 0.029 & 0.029 & 0.029 & 0.029 & 0.029 & 0.029 & 0.029 & 0.028 \\
\hline
\end{tabular}




\section{References}

Fabozzi, F. J., Ma, K., \& Oliphant, B. J. (2008). Sin stock returns. Journal of Portfolio Management, 35, 82-94.

Hong, H., \& Kacperczyk, M. (2009). The price of sin: The effects of social norms on markets. Journal of Financial Economics, 93, 15-36. 